\title{
Scale-by-scale analysis of probability distributions for global MODIS-AQUA cloud properties: how the large scale signature of turbulence may impact statistical analyses of clouds
}

\author{
M. de la Torre Juárez, A. B. Davis, and E. J. Fetzer \\ Jet Propulsion Laboratory/California Institute of Technology, Pasadena, CA 91109-8099, USA \\ Received: 21 August 2010 - Published in Atmos. Chem. Phys. Discuss.: 7 September 2010 \\ Revised: 22 February 2011 - Accepted: 3 March 2011 - Published: 28 March 2011
}

\begin{abstract}
Means, standard deviations, homogeneity parameters used in models based on their ratio, and the probability distribution functions (PDFs) of cloud properties from the MODerate resolution Infrared Spectrometer (MODIS) are estimated globally as function of averaging scale varying from 5 to $500 \mathrm{~km}$. The properties - cloud fraction, droplet effective radius, and liquid water path - all matter for cloud-climate uncertainty quantification and reduction efforts. Global means and standard deviations are confirmed to change with scale. For the range of scales considered, global means vary only within $3 \%$ for cloud fraction, $7 \%$ for liquid water path, and $0.2 \%$ for cloud particle effective radius. These scale dependences contribute to the uncertainties in their global budgets. Scale dependence for standard deviations and generalized flatness are compared to predictions for turbulent systems. Analytical expressions are identified that fit best to each observed PDF. While the best analytical PDF fit to each variable differs, all PDFs are well described by log-normal PDFs when the mean is normalized by the standard deviation inside each averaging domain. Importantly, log-normal distributions yield significantly better fits to the observations than gaussians at all scales. This suggests a possible approach for both sub-grid and unified stochastic modeling of these variables at all scales. The results also highlight the need to establish an adequate spatial resolution for two-stream radiative studies of cloud-climate interactions.
\end{abstract}

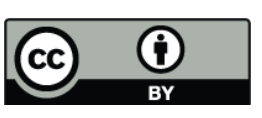

Correspondence to:

M. de la Torre Juárez

(mtj@jpl.nasa.gov)

\section{Introduction}

Cloud impacts on the energy and water cycles remain an important source of uncertainty in our understanding of climate. This applies to the simplest low-dimensional energy balance models (Budyko, 1969; Sellers, 1969; Pujol, 2003), climate sensitivity analyses (e.g. Roe and Baker, 2007; Hannart et al., 2009), two-scale stochastic models (e.g. Imkeller and v. Storch, 2001; Díaz et al., 2009), and to state of the art Global Circulation Models (GCMs) incorporated into the Intergovernmental Panel on Climate Change assessments (Solomon, 2007). The inherent turbulence of atmospheric flows prevents observations and models from capturing the constantly evolving structure of clouds in the atmosphere. This complexity limits our confidence in predictions of cloud properties and therefore of climate sensitivity. Observed cloud properties have biases dependent on sensor- (e.g. Boers et al., 2006; Horváth and Davies, 2007; Bennartz, 2007) and cloud-types (de la Torre Juárez et al., 2009) that may be smaller than those resulting from limited sampling of highly variable fields (Schutgens and Roebeling, 2009). Therefore, one approach to understand the radiative impact of clouds on climate is to determine the robust statistical distributions of cloud properties rather than solving exactly for each specific cloud field. Although it is widely recognized that there is no justification for assuming gaussian distributions (Hannart et al., 2009), analyses of atmospheric flows and climate often quantify cloud-climate dynamics and uncertainties by interpreting means, standard deviations and confidence levels in gaussian frameworks (e.g. Roe and Baker, 2007). Identifying more realistic distributions gives more credible depictions of observational results, better subgrid parameterizations, and a more rigorous approach to quantifying cloud and climate modeling uncertainties.

Published by Copernicus Publications on behalf of the European Geosciences Union. 

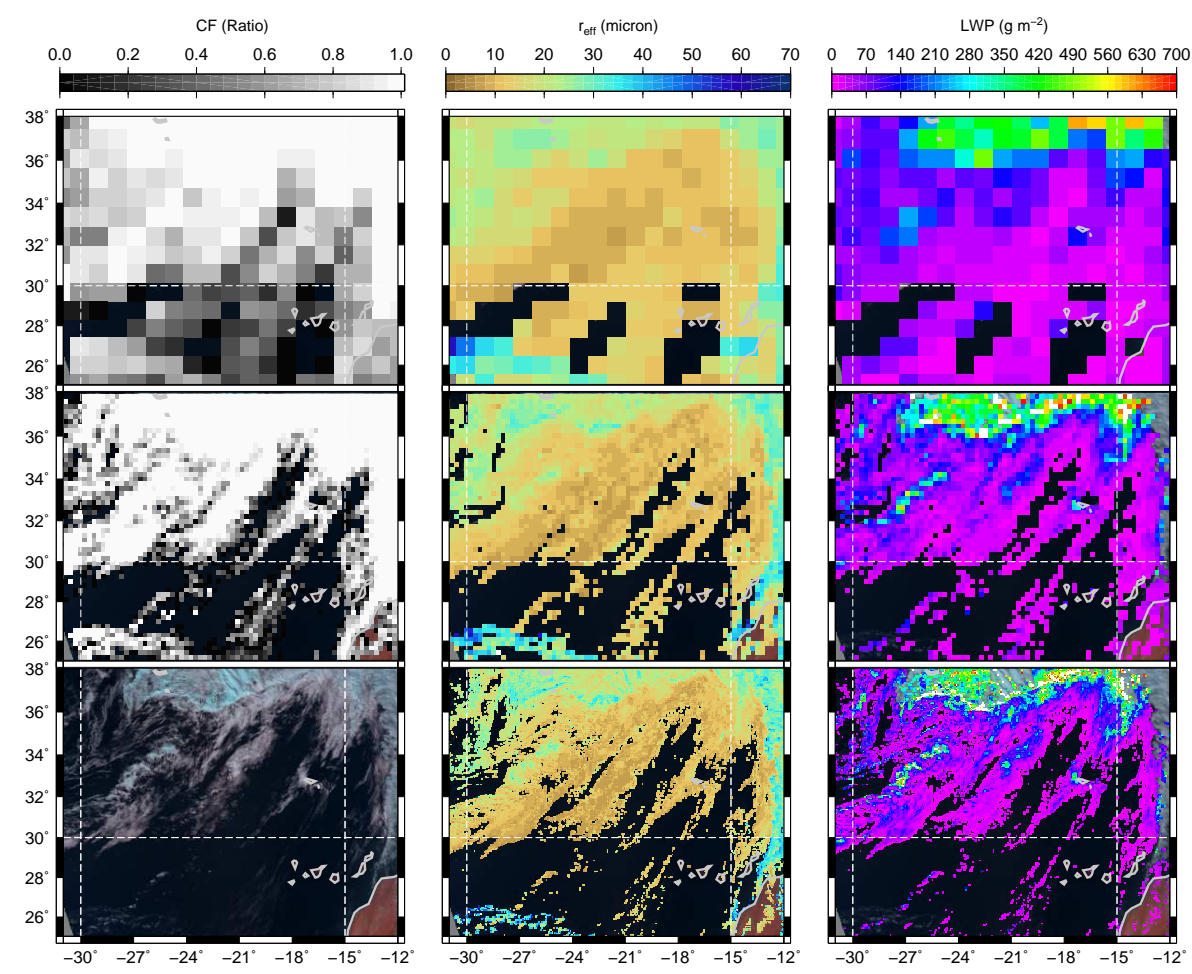

Fig. 1. Cloud fraction (CF), cloud droplet effective radius $\left(r_{\text {eff }}\right)$ and cloud liquid water path (LWP) for high and low clouds W and NW of the Canary Islands at spatial resolutions of $5 \mathrm{~km}, 25 \mathrm{~km}$ and $100 \mathrm{~km}$. The lower left-hand panel is a $1 \mathrm{~km}$ resolution with a three-color rendering of the scene using three MODIS L1B bands: Band 3 (459-479 nm), Band 4 (545-565 nm), and Band 5 (1230-1250 nm). The figure emphasizes the multi-layered nature of the cloudiness. Different spatial resolutions suggest different statistics of cloud properties.

Questions also remain open about climate impacts of processes unfolding at the relatively small scales of the clouds themselves. The spatio-temporal scales at which cloud formation, precipitation, and interactions with aerosols occur are largely unresolved by satellite instruments, yet these phenomena determine large-scale properties of clouds relevant to the atmosphere's radiative balance. The ability to characterize statistically a large range of scales can reveal dynamical interactions across scales, and, possibly, to extrapolate these to the small unresolved ones, thus providing relevant validation data for cloud-process models.

Comparisons of trade cumulus cloud fraction statistics over the tropical Western Atlantic at pixel resolutions from the $15-\mathrm{m}$ to the kilometer scale show significant scaledependence (e.g. Dey et al., 2008). Similar scale-dependence was found in early data-driven stochastic simulations of cloud fields (Shenk and Salomonson, 1972), in observed outgoing longwave radiation in high tropical Pacific clouds (Pierrehumbert, 1996), in albedo from optical depths (Oreopoulos and Davies, 1998), in global cloud optical thickness, emissivity and cloud top temperature (Barker et al., 1996; Rossow et al., 2002), and in liquid water path of lowlevel marine clouds over the Pacific (Wood and Hartmann, 2006). These studies showed that different averaging scales result in apparent biases between instruments, and between instruments and models. This effect is illustrated in Fig. 1 where cloud properties are shown at different resolution scales of $5 \mathrm{~km}, 25 \mathrm{~km}$ and $100 \mathrm{~km}$ for the same cloud scene. Yet analyses of global cloud variables and climate properties occur typically at far coarser scales. For instance, the global radiative budget of the atmosphere has been studied at $10^{\circ} \times 10^{\circ}$ (e.g. Forster and Gregory, 2006); satellite-based observational studies of the hydrological cycle are found at resolutions of $1^{\circ} \times 1^{\circ}$ (Schlosser and Houser, 2007); studies of the global radiative balance from weather analyses are often at resolutions of $2.5^{\circ} \times 2.5^{\circ}$ (Trenberth et al., 2003); global effects of aerosols on clouds driven by micro-scale interactions are modeled at $5^{\circ} \times 5^{\circ}$ and $2.5^{\circ} \times 2.5^{\circ}$ resolutions (Quaas et al., 2009, and references therein). At the other end of the spectrum, some satellites such as LandSat can provide cloud information at scales of a few meters (Dey et al., 2008), and airborne instruments measure cloud properties at even smaller scales.

This paper compares satellite-based inferences of a set of cloud properties relevant to cloud-climate interactions, and looks for the best fit analytical probability distribution functions (PDFs). The properties are: Cloud Fraction (CF), which modulates the amount of radiation reaching the surface and how much is reflected back into space; cloud liquid water path (LWP), which acts as a powerful barrier 
a)

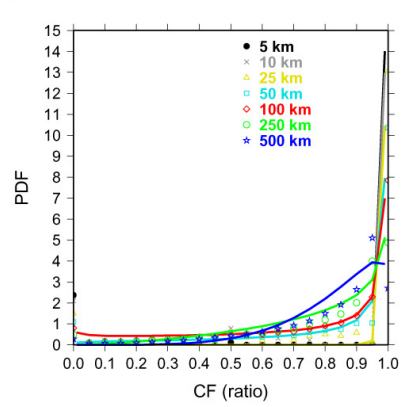

d)

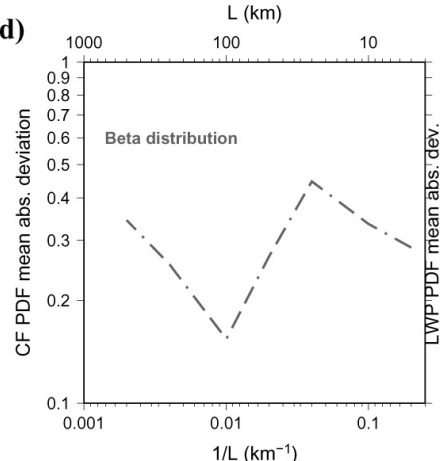

b)

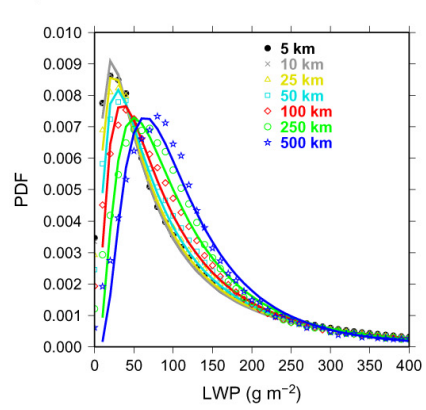

e)

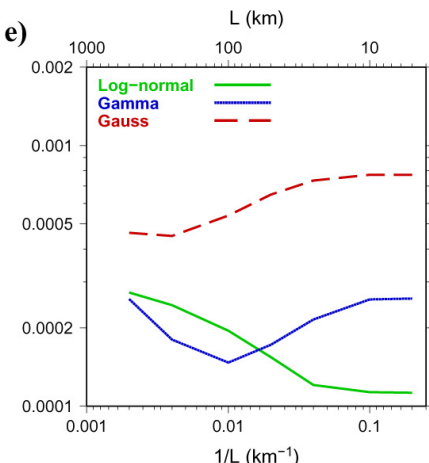

c) $\quad r_{\text {eff }}$ Fit to Gamma distribution.
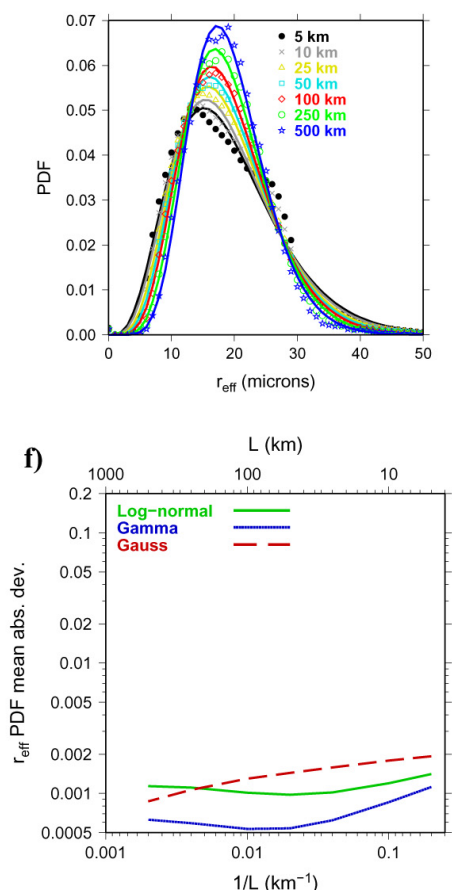

Fig. 2. (a-c) Symbols show the observed values at different spatial averaging scales. Solid lines are the designated least-squares fits. All PDFs for all variables are non-symmetric. LWP and $r_{\text {eff }}$ have respectively log-normal and exponential tails that capture the infrequent high values. (d-f) Mean absolute deviations between the observed global CF (d), LWP (e), $r_{\text {eff }}$ (f) and the analytical PDFs after a nonlinear least-squares fit.

of outgoing radiation; and cloud particle effective radius $\left(r_{\text {eff }}\right)$, which modulates the radiative absorption properties of clouds and the Earth's albedo. LWP is derived from $r_{\text {eff }}$ and cloud optical depth, $\tau$, through LWP $\propto \rho_{\mathrm{w}} \tau r_{\text {eff }}$, with $\rho_{\mathrm{W}}$ the condensed water density (Platnick et al., 2003). Analytical PDFs fitted here, besides gaussians, have been proposed before: beta for CF (Falls, 1974), Gamma for LWP and $\tau$ (Newman et al., 1995; Barker et al., 1996), and log-normal for turbulent processes (Monin and Yaglom, 1975). PDFs are for global CF, $r_{\mathrm{eff}}$ and LWP, and means and standard deviations are estimated at resolutions from 5 to $500 \mathrm{~km}$ using Collection 5 retrievals from the MODerate resolution Infrared Spectrometer (MODIS) aboard the AQUA spacecraft (King et al., 2006).

We quantify the scale-dependence of statistical moments and compare them to predictions of generalized flatness scaling for self-similar homogeneous turbulent flows (Monin and Yaglom, 1975; Frisch, 1995). Quantitative empirical evidence for the turbulent nature of clouds from a space-based perspective goes at least back to Lovejoy (1982), who invoked fractal geometry, and continues to come using multifractal statistics (Lovejoy et al., 2009). To the best of our knowledge, previous studies of satellite observations have used raw (Level-1/radiance) data, and gradients thereof in the case of multifractal analysis, while here we use retrieved (standard Level-2) cloud properties. It is reassuring (Davis et al., 1994) to see that, in spite of all the assumptions used to process radiances into standard cloud products, the signature of turbulence is still clear and can be represented simply enough for practical parameterization of cloud processes in climate models.

\section{Analytical PDFs at different scales}

Figure 2a-c shows global PDFs for CF, $r_{\text {eff }}$, and LWP observed over 10 equinox days in 2003 to 2007 at seven spatial resolutions. Equinox days minimize possible seasonal biases while handling the large amount of high spatial resolution data needed to cover a significant number of years. The data are from five-minute granules of MODIS-AQUA daytime observations. A granule covers about $1354 \times 2030 \mathrm{~km}^{2}$ and is treated as if it were a "realization" of a cloud experiment. Cloud fraction was considered where CF and Cloud Top Pressure (CTP) were flagged as useable. LWP and $r_{\text {eff }}$ are for the same clouds if the LWP is flagged by MODIS as useable. CTP flags were checked to limit the differences in cloud populations from this study to those that discriminate cloud heights. Data confidence levels were allowed to be marginal, good and very good. This assumes that marginal retrievals can return instantaneously incorrect but plausible 
values. Cloud properties (CF, LWP, $\left.r_{\text {eff }}, \mathrm{CTP}\right)$ were calculated by averaging each variable over basic spatial domains, that we will call "pixels", of side length $L$ and then calculating the global average and standard deviation of all those local means for each granule. We used seven different pixel resolutions: $L=5,10,25,50,100,250$, and $500 \mathrm{~km}$. No constraints were set on the percentage of valid retrievals in each scene, their CTP, or if the clouds were over land or ocean. The resulting number of 5-min granules for these 10 days were 2880 realizations. Analytical PDFs were fitted at all spatial resolutions to the observed CF, LWP and $r_{\text {eff }}$ distributions using a non-linear least-squares approach with two fitting parameters related to the mean and standard deviation.

As expected (Falls, 1974), the observed bimodal PDF of $\mathrm{CF}$ is best fit to a beta distribution. Bimodal beta functions could be found that fit to the bimodal observations (see symbols accumulating at $\mathrm{CF}=0$ and $\mathrm{CF}=1$ in Fig. 2a), but the better fit to our dataset occured at the parameters for a nonbimodal beta distribution (solid lines in Fig. 2a). The CF is the $5 \mathrm{~km}$ resolution MODIS cloud product where the only possible values are 1 and 0 (overcast or clear). Because our gridding is fixed to location and cloud structures are not, an observed 5-km cloud structure may touch over one, two, or up to four $5-\mathrm{km}$ grid cells and appear as two cells with a $\mathrm{CF}=0.5$ or four contiguous cells with $\mathrm{CF}=0.25$. The impact of this effect is visible in Fig. 2a where some CF values at $5 \mathrm{~km}$ are $0.5,0.25$ and 0.75 . Figure $2 \mathrm{a}$ also shows that such cases are few and have little statistical impact. As the spatial resolution is degraded, this effect may still happen at the perimeter of the larger cells but their proportion and statistical impact compared to those within a larger area decays with increasing $L$. As the spatial resolution is degraded, Fig. 2a shows that a continuum of values emerges through averaging clear and overcast scenes onto one mean value. At the coarser resolutions of $250 \mathrm{~km}$ and above, the higher peak shifts from 1 towards 0.9 . This scale-dependent behavior of $\mathrm{CF}$ is consistent with that found for clear scenes using the MODIS 1-km cloud mask (Krijger et al., 2007) where confidence levels (confident cloudy, probably cloudy, probably clear, confident clear) were translated into percentages of clear sky at $1 \mathrm{~km}$ and lower spatial resolutions.

Unlike CF, LWP and $r_{\text {eff }}$ have a (theoretically) unbounded range of values. Both show skewed, hence non-gaussian, distributions in Fig. 2. Figure 2d-f compares the minimum absolute deviation between observed PDF and least mean squares fit to a beta, gamma, log-normal, and gaussian distributions at the different pixel sizes to find that the lognormal, a popular choice in turbulence studies, is a better choice for LWP at resolutions finer than $100 \times 100 \mathrm{~km}^{2}$, and the Gamma function is better at coarser resolutions (Fig. 2e). Gamma PDFs provide the best fit to $r_{\text {eff }}$ at all scales (Fig. 2f), which is consistent with how $r_{\text {eff }}$ depends on cloud droplet radius (Pointikis and Hicks, 1992) and how droplet radius follows Weibull/Gamma distributions (Liu and Hallet, 1998). Figures $2 \mathrm{e}$ and $\mathrm{f}$ show that the peak (mode) and tails of LWP and $r_{\text {eff }}$ PDFs change with spatial resolution. Large deviations determine the tails on LWP and $r_{\text {eff }}$ distributions in these figures. As the spatial resolution is decreased, the averages over larger areas blur these extreme values. As a result, the distributions at coarser resolutions appear more symmetric, the means shift closer to the peaks of the distributions and the tails shorten.

\section{Scale dependence of statistical moments}

Figure 3 shows the scale dependence of global (from all granules in our study) statistical moments for pixel averaged, LWP and $r_{\text {eff. }}$ The moments in Fig. 3 all change as a function of scale. The normalized mean, a frequent diagnostic in turbulence studies is also shown. It is the inverse of the global "relative dispersion" in Pointikis and Hicks (1992); and the square root of the global "homogeneity parameter" in Wood and Hartmann (2006). The normalized mean enables to compare the relevance of the scale-dependence for variables with different values. Numerical values in Table 1 show mean CF and LWP more scale-dependent than $r_{\text {eff. At }}$ the same time, the normalized mean (and the dispersion) for $r_{\text {eff }}$ changes over a factor two.

Kostinski and Shaw (2001) argued that cloud particle aggregations at microphysics scales obey statistics similar to those of binary-valued fields with auto-correlation functions decaying as scale increases. An exponential decay reveals a discrete Poisson distribution of cloudy-clear interfaces. If $\mathrm{CF}$ is decorrelated at $5 \mathrm{~km}$ (as in Schutgens and Roebeling, 2009), then consecutive sampling of CF from uncorrelated pixels is analogous to a temporal sampling of a random binary (cloudy-clear) outcome, time being proportional to the number of pixels sampled. Therefore, if CF statistics follow such Poisson-type rules and self-similarity holds up to 5-km resolution, the absolute deviation would approach the mean value. This is not seen in Fig. 3a where mean CF over its standard deviation decreases with increasing pixel size but remains above unity for all the range 5 to $500 \mathrm{~km}$.

Normalized mean LWP approaches unity at the $100 \times 100 \mathrm{~km}^{2}$ pixel size, despite LWP not being a bimodal distribution. A possible explanation could come from arguments similar to those from turbulence theory (Frisch, 1995) where the scale-dependence of statistical moments for a zero-centered variable, $X$, gives information about how its variance is transferred across scales in turbulent flows. Following Jiménez (2007), we define a generalized structure function of order $n$ as: $S_{X}(n)=\int X^{n} P(X) \mathrm{d} X, P(X)$ being its PDF. $S_{X}(n)$ is used to define a generalized flatness factor as $Y_{X}(n)=S_{X}(n) / S_{X}(2)^{n / 2}$. Kolmogorov's self-similarity hypothesis for homogeneous turbulence ( $X$ is velocity $v$ ) leads to the scaling law $S_{v}(n) \sim L^{n / 3}$, at least for low $n$, and thus $Y_{v}(n)$ is independent of $L$. Note that the normalized LWP means in Fig. $3 \mathrm{~b}$ are 

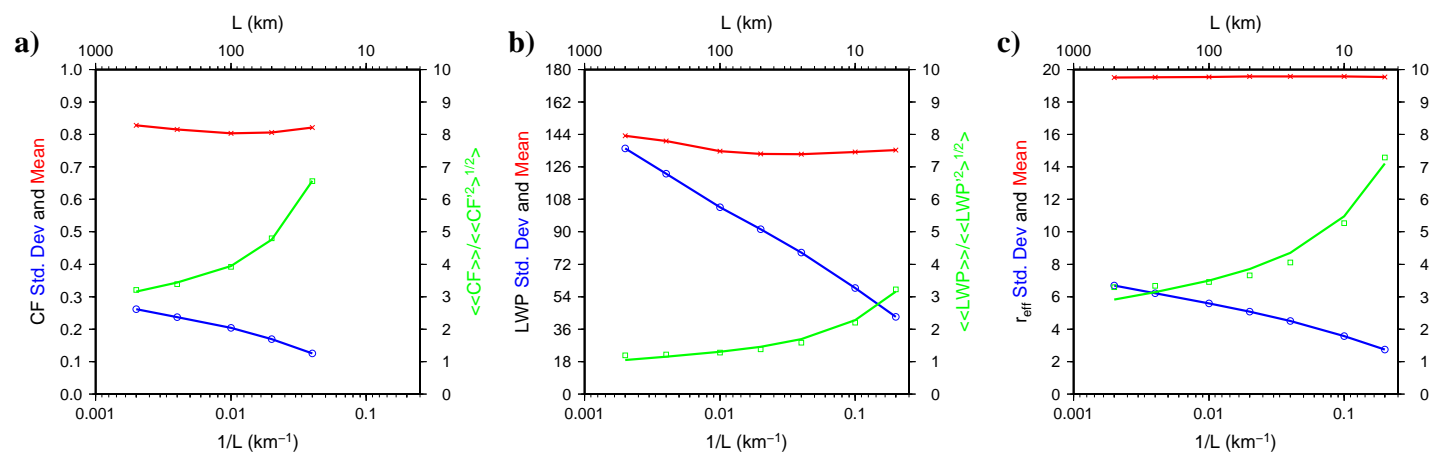

Fig. 3. Scale dependence of the global mean (red), global standard deviations (blue), and mean rescaled over the global standard deviation (green) for CF, LWP and $r_{\text {eff }}$. The double brackets $\ll \cdots \gg$ stand for "global/ensemble average of local means (at a specific scale)". The primed quantities on the green y-axis refer to deviations from the global average value.

Table 1. Observed means, standard deviations and dispersions (inverse of the normalized means) in the panels of Fig. 3. Parentheses show the $\%$-difference of every mean from the mean of all means, i.e., the global average.

\begin{tabular}{llccccccc}
\hline Variable & Moment & $5 \mathrm{~km}$ & $10 \mathrm{~km}$ & $25 \mathrm{~km}$ & $50 \mathrm{~km}$ & $100 \mathrm{~km}$ & $250 \mathrm{~km}$ & $500 \mathrm{~km}$ \\
\hline \multirow{2}{*}{$\mathrm{CF}$} & Mean & - & - & $0.82(0.8 \%)$ & $0.81(-1.1 \%)$ & $0.80(-1.4 \%)$ & $0.82(0.1 \%)$ & 0.83 \\
& Standard & - & - & 0.12 & 0.17 & 0.20 & 0.24 & 0.26 \\
& Dispersion & - & - & 0.15 & 0.21 & 0.25 & 0.29 & 0.32 \\
\hline \multirow{2}{*}{ LWP $\left(\mathrm{g} \mathrm{m}^{-2}\right)$} & Mean & $135.28(-0.7 \%)$ & $134.20(-1.5 \%)$ & $133.00(-2.4 \%)$ & $133.18(-2.3 \%)$ & $134.79(-1.1 \%)$ & $140.38(3.0 \%)$ & $143.22(5.1 \%)$ \\
& Standard & 42.8 & 58.8 & 78.5 & 91.5 & 103.7 & 122.3 & 136.2 \\
& Dispersion & 0.32 & 0.44 & 0.59 & 0.69 & 0.77 & 0.87 & 0.95 \\
\hline \multirow{2}{*}{$\mathrm{r}_{\text {eff }}(\mu \mathrm{m})$} & Mean & $19.54(0.1 \%)$ & $19.57(0.1 \%)$ & $19.57(0.1 \%)$ & $19.57(0.0 \%)$ & $19.54(-0.1 \%)$ & $19.52(-0.1 \%)$ & $19.51(-0.1 \%)$ \\
& Standard & 2.75 & 3.57 & 4.50 & 5.08 & 5.59 & 6.21 & 6.70 \\
& Dispersion & 0.14 & 0.18 & 0.23 & 0.26 & 0.29 & 0.32 & 0.34 \\
\hline
\end{tabular}

$$
Y_{\mathrm{LWP}}(1)=\frac{\langle\langle\mathrm{LWP}\rangle\rangle_{\text {global }}}{\left\langle\langle\mathrm{LWP}\rangle^{2}-\langle\langle\mathrm{LWP}\rangle\rangle_{\text {global }}^{2}\right\rangle_{\text {global }}^{1 / 2}}
$$

and note that in turbulence $X$ is a centered (zero-mean) random variable. The scaling in Fig. $3 b$ for LWP at pixel sizes of $100 \times 100 \mathrm{~km}^{2}$ and larger is then consistent with that of selfsimilar turbulent structure functions for flows in the inertial subrange.

The observed $Y_{\mathrm{CF}}(1)$ and $Y_{r_{\mathrm{eff}}}(1)$ do not converge to unity in Fig. 3a, but approach a linear law in the inverse pixel side length, $1 / L$. A linear fit, $\tilde{Y}_{\mathrm{CF}}=3.03+88.21 / L$ was found with mean absolute deviation $\Delta_{\mathrm{CF}}=100 \times\langle| 1-$ $\tilde{Y}_{\mathrm{CF}}(1) / Y_{\mathrm{CF}}|\rangle=0.9 \%$. Similar fits to the global normalized means for LWP and $r_{\text {eff yield }} \tilde{Y}_{\text {LWP }}=1.18+10.26 / L$ and $\tilde{Y}_{r_{\text {eff }}}=3.25+20.17 / L$ respectively, with larger mean absolute deviations $\Delta_{\mathrm{LWP}} \approx \Delta_{r_{\mathrm{eff}}}=5.5 \%$. As expected from a turbulence perspective, global means change far less than standard deviations when looking for power laws in $L$. Specifically, we find $\langle\mathrm{CF}\rangle=0.8 L^{0.004}(\Delta=1 \%),\langle\mathrm{LWP}\rangle=$ $124 L^{0.013}(\Delta=1.6 \%)$, and $\left\langle r_{\text {eff }}\right\rangle=19.6 L^{-0.001}(\Delta=0.1 \%)$, while $\left\langle\mathrm{CF}^{2}\right\rangle^{1 / 2}=0.06 L^{0.24}(\Delta=5.6 \%) \quad\left\langle\mathrm{LWP}^{\prime 2}\right\rangle^{1 / 2}=$ $33 L^{0.24}(\Delta=6.6 \%),\left\langle r_{\text {eff }}^{\prime 2}\right\rangle^{1 / 2}=2.3 L^{0.18}(\Delta=6.1 \%)$, which approach $L^{1 / 4}$.

\section{PDFs of locally normalized means}

In essence, Fig. 4 shows statistics of statistics as a function of scale. The PDFs are for means over each pixel normalized locally by the standard deviation over all observations within the pixel. Because $\mathrm{CF}$ is given at $5 \times 5 \mathrm{~km}^{2}$, a minimum of $25 \times 25 \mathrm{~km}^{2}$ is necessary for the CF pixels to accumulate some significant standard deviation. The notable finding is that, while the global PDFs of CF, LWP, and $r_{\text {eff }}$ display different functional forms in Fig. 2, the global PDFs of locally normalized means have a very similar shape for all variables and all are fitted best by log-normal distributions. Notice that the PDFs have been displayed in log-scales making the tails more visible and, as is often seen in turbulence, they appear to be power-law. However, they contribute little to the absolute deviation from the fit when weighted by their frequency of occurrence. Indeed, weighting the absolute deviations by the observed value (thin lines in Fig. 5a-c) measures the deviation from the functional shape, and this shows that the log-normal remains best for LWP and $r_{\text {eff }}$ at all domain sizes and it worsens for $\mathrm{CF}$ at resolutions finer than $250 \times 250 \mathrm{~km}^{2}$. 
a) Normalized CF PDF and Log-Normal fit.

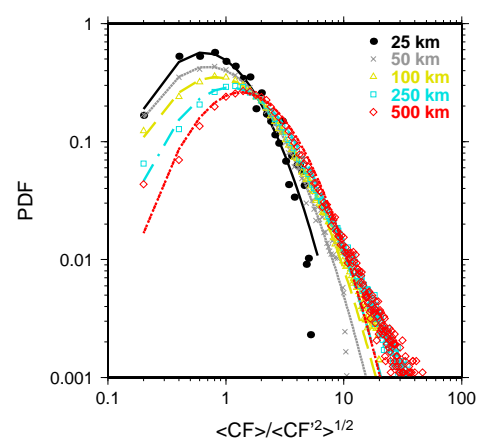

b) Normalized LWP PDF and Log-Normal fit.

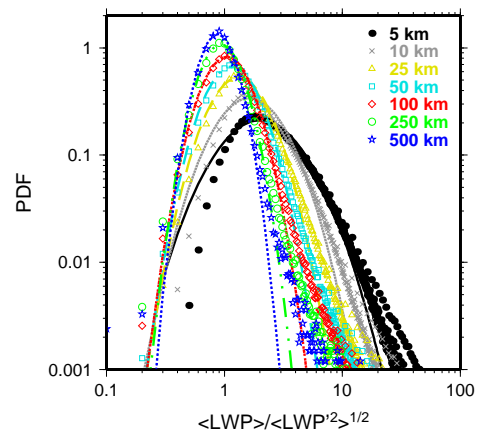

c) Normalized $r_{\text {eff }}$ PDF and Log-Normal fit.

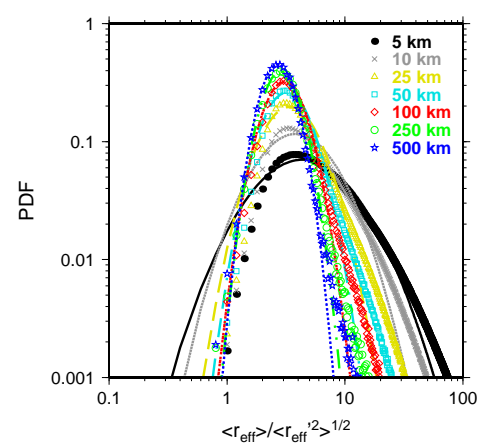

Fig. 4. A different look into cloud property PDFs: means normalized by standard deviations as a function of scale.
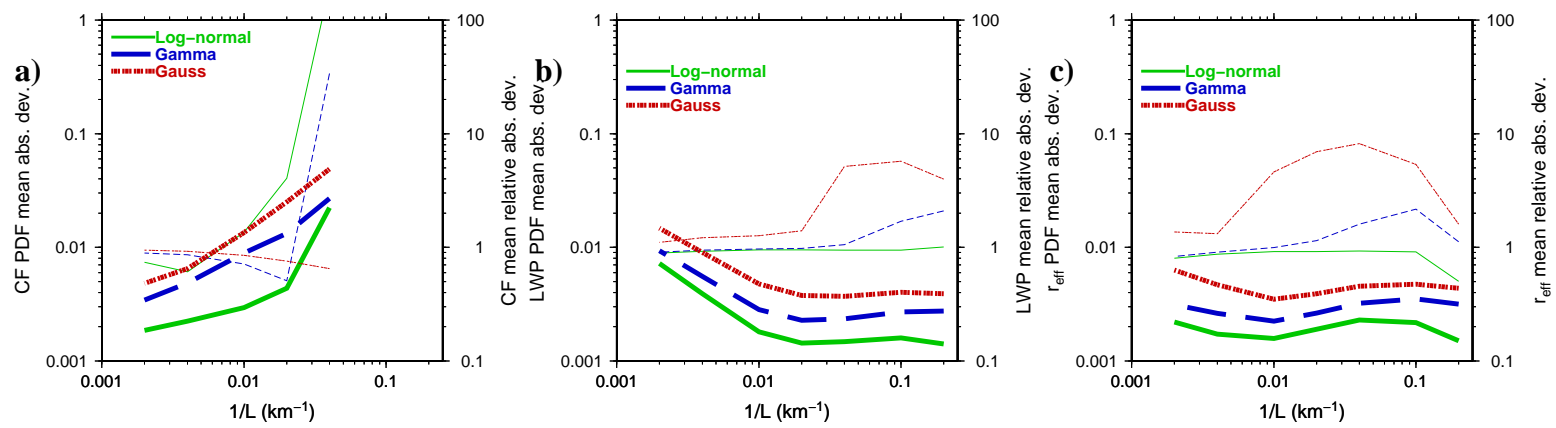

Fig. 5. Mean absolute deviations between the observed and the analytical PDFs in thick lines; thin lines show the mean absolute deviation between fit and observations when weighted by the inverse of the observed PDF at each point.

\section{Summary and conclusions}

Notwithstanding MODIS measurement errors (Boers et al., 2006; Horváth and Davies, 2007; Bennartz, 2007; de la Torre Juárez et al., 2009) and those biases caused by incomplete sampling of highly variable fields (Oreopoulos et al., 2009; Schutgens and Roebeling, 2009), this study has intercompared a set of analytical functions that best fit the observed PDFs of global macroscopic cloud properties across a large range of scales. Observed cloud fraction is best approached by beta distributions, droplet effective radius by a Gamma PDF, and liquid water path follows closely a lognormal or a Gamma distribution. Gaussian PDFs are never the best description.

The global normalized mean CF decreases linearly with the size $L$ of the local averaging domain down to about $100 \times 100 \mathrm{~km}^{2}$ areas, at which point it trends upward to a resolution of $500 \times 500 \mathrm{~km}^{2}$. Average LWP changes little from $10 \times 10 \mathrm{~km}^{2}$ to $100 \times 100 \mathrm{~km}^{2}$ where it starts increasing linearly with $1 / L$. Globally averaged $r_{\text {eff }}$ seems to be independent of the spatial resolution. A scale-independent average $r_{\text {eff }}$ is consistent with predictions of a droplet size saturation scale based on entropy maximizing size distribu- tions of cloud droplets (Liu et al., 2002) in a turbulent atmosphere. However, normalized means of $r_{\text {eff }}$ change more significantly, with a linear dependence on $1 / L$ and this is a factor to take into account when modeling cloud distributions using a homogeneity parameter.

The mode of the finer resolution $\mathrm{CF}$ and the coarser LWP distributions approach unity, which is consistent with the domain-level statistics following self-similar scaling: $Y_{\mathrm{LWP}}(1) \sim$ constant, as described earlier, in analogy with turbulence theory and observations of zero-centered variables. This similarity raises the possibility of applying some of the general scaling arguments used in turbulent flows to describe LWP scale dependence. Notice that, for instance, the beta-model, multifractal, and other approaches discussed in the context of turbulent flows (e.g. Frisch, 1995, chapter 8) rely only on how characteristic time scales and objects sizes affect the structure functions at different averaging lengths. Exploring such random scaling models is of interest but would require testing predictions for higher order structure functions and moments and becomes a task for future efforts.

Other results include a test for possible connections to selfsimilar Poissonian statistics for $\mathrm{CF}$, which fails at $5 \mathrm{~km}$ and 
LWP Fit to Log-Normal distribution.

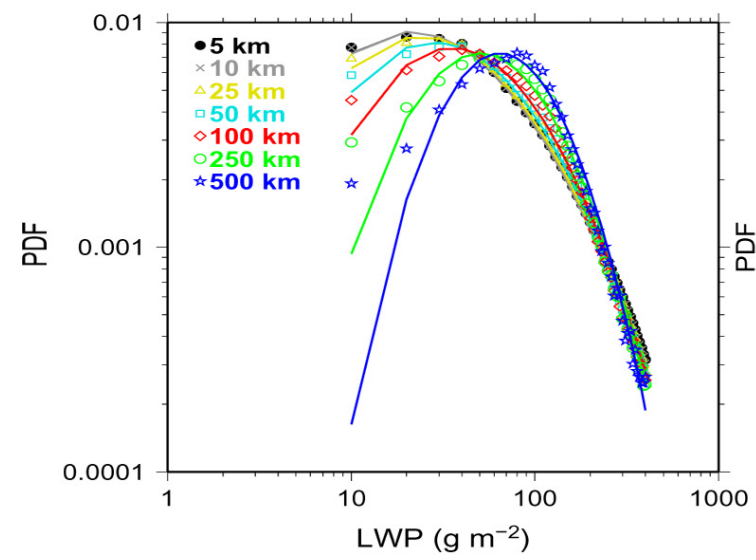

$r_{\text {eff }}$ Fit to Gamma distribution.

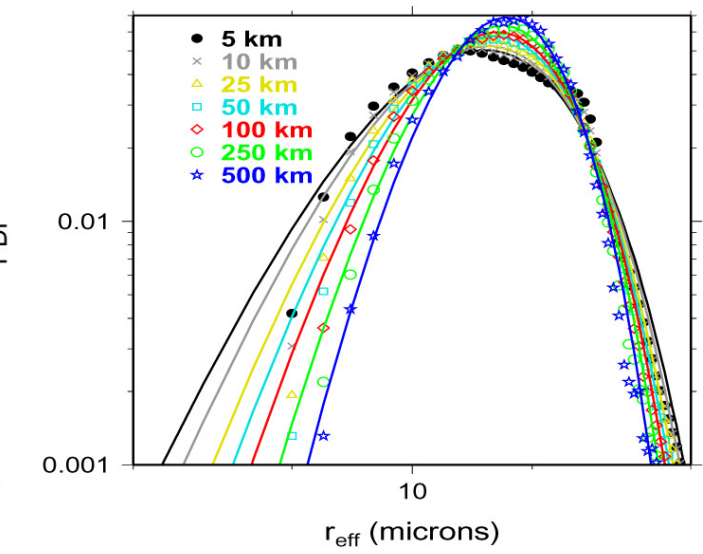

Fig. 6. PDFs for LWP and $r_{\text {eff }}$ in logarithmic scale highlight the increasing LWP anomaly at the low end of the log-normal distribution.

above. Finally, on the scale dependence, power laws are found for means as a function of pixel size and they behave differently from standard deviations, with CF and LWP standard deviations approaching $L^{1 / 4}$, as is familiar in turbulent flows.

Shell-models of turbulence and the entropy maximization models of $r_{\text {eff }}$ describe PDFs at the micro-scale, a link still needs to be established to scales more relevant for climate and cloud-process models used in this work, $\sim \mathrm{km}$ and above. Scale-by-scale analysis provides a step in that direction. For instance, the cloud droplet radius models of Liu and Daum (2000) provide a probability $p(r)$, which leads to an effective radius $r_{\text {eff }}=\left\langle r^{3}\right\rangle /\left\langle r^{2}\right\rangle=\int r^{3} p(r) \mathrm{d} r / \int r^{2} p(r) \mathrm{d} r$. In their model, $r_{\text {eff }} \sim \alpha(\mathrm{LWC} / N)^{1 / 3}$ where LWC is the liquid water content (i.e., LWP/cloud thickness in stratus), $N$ the total droplet number concentration in a parcel of air, and $\alpha$ a parameter related to the heterogeneity of the sampled cloud parcel. Our study, which provides the global scale-dependent probability distributions, presents $\operatorname{PDF}_{L}\left(r_{\text {eff }}\right)$ where $L$ is scale and the random variable is the MODIS pixel-scale retrieved effective radius averaged over scale $L$, which we can interpret as $\operatorname{PDF}_{L}\left[\alpha(\mathrm{LWC} / N)^{1 / 3}\right]$ with the same averaging. $\mathrm{PDF}_{L}\left(r_{\text {eff }}\right)$ becomes a measure of the fluctuations of the parameters in the micro-scale probability distributions $\alpha$ and $\mathrm{LWC} / N$, which will depend on the type(s) of cloud present in an area of sidelength $L$.

We have presented the means and PDFs of locally normalized mean CF, LWP and $r_{\text {eff }}$ (mean over standard deviation inside pixels of a given size) that measure the heterogeneity of clouds within each pixel and found that they follow a scale-dependent log-normal distribution for all three variables, thus providing a possible unified description of these cloud properties at all scales in climate model parameterizations of sub-grid processes. Still, the normalized PDFs have tails associated with extreme values and unusually low variability missed by the closest log-normal or Gamma. If a relative weighting is used to measure the distance from the empirical histogram shape, the normalized $\mathrm{CF}$ at resolutions higher than $250 \times 250 \mathrm{~km}^{2}$ is better approached by a Gamma distribution.

The scale dependence of cloud variability highlights that care is needed to choose a spatial resolution for analyses of global cloud-radiative effects. Rossow et al. (2002) argue that significant horizontal radiative transfer at scales below $3 \mathrm{~km}$ justify considering cloud properties at scales only above $3 \mathrm{~km}$ for global analyses based on two-stream (up-down) radiative models. This hypothesis may be tested by looking at the radiative impacts of clouds with different sizes and cloud fractions at small scales. Since MODIS CF and $\tau$ statistics over $5 \times 5 \mathrm{~km}^{2}$ regions are expected to differ little from the values at $3 \mathrm{~km}$ (Dey et al., 2008), $5 \mathrm{~km}$ would be a good choice for such a future analysis.

Another effect observed in in this study and highlighted in Fig. 6 is that lower LWP values deviate significantly from the log-normal distribution values as pixel resolution is graded. The oposite effect is observed on $r_{\text {eff }}$. This departure on LWP from the analytical distribution captures the relative frequency of scenes with medium and thin clouds becoming stronger when $L>50 \mathrm{~km}^{2}$, but their influence on the global mean LWP is masked by the contribution by the tail with extremely high LWP values that seem to dominate the global average and shift it towards higher means at $L>100 \mathrm{~km}^{2}$.

Acknowledgements. Funding provided by the NASA MEaSURES and NEWS programs. The work was carried out at the Jet Propulsion Laboratory/California Institute of Technology, under a contract with the National Aeronautics and Space Administration. Conversations with J. Teixeira and J. Jiménez, and comments by the anonymous reviewers are gratefully acknowledged. MODIS data are from http://ladsweb.nascom.nasa.gov/. Numpy, Scipy modules and the Generic Mapping Tools (SOEST-GMT) were used for this work.

Edited by: T. Garrett 


\section{References}

Barker, H., Wielicki, B., and Parker, L.: A parameterization for Computing Grid-Averaged Solar Fluxes for Inhomogeneous Marine Boundary Layer Clouds, Part II: Validation using satellite data., J. Atmos. Sci., 53, 2304-2316, 1996.

Bennartz, R.: Global assessment of marine boundary layer cloud droplet number concentration from satellite, J. Geophys. Res., 112, D02201, doi:10.1029/2006JD007547, 2007.

Boers, R., Acarreta, J. R., and Gras, J. L.: Satellite monitoring of the first indirect aerosol effect: Retrieval of the droplet concentration of water clouds, J. Geophys. Res., 111, D22208, doi:10.1029/2005JD006838, 2006.

Budyko, M.: The effect of solar radiation variations on the climate of the Earth, Tellus, 21, 611-619, 1969.

Davis, A., Marshak, A., Wiscombe, W., and Cahalan, R.: Multifractal characterizations of non-stationarity and intermittency in geophysical fields: Observed, retrieved, or simulated, J. Geophys. Res., 99, 8055-8072, 1994.

de la Torre Juárez, M., Kahn, B. H., and Fetzer, E. J.: Cloudtype dependencies of MODIS and AMSR-E liquid water path differences, Atmos. Chem. Phys. Discuss., 9, 3367-3399, doi:10.5194/acpd-9-3367-2009, 2009.

Dey, S., Girolamo, L. D., and Zhao, G.: Scale effect on statistics of the macrophysical properties of trade wind cumuli over the tropical western Atlantic during RICO, J. of Geophys. Res., 113, D24214, doi:10.1029/2008JD010295, 2008.

Díaz, J., Langa, J., and Valero, J.: On the asymptotic behaviour of solutions of a stochastic energy balance climate model, Physica D, 238, 880-887, 2009.

Falls, L. W.: The Beta Distribution: A Statistical Model for World Cloud Cover, J. Geophys. Res., 79, 1261-1264, 1974.

Forster, P. M. F. and Gregory, J. M.: The Climate Sensitivity and Its Components Diagnosed from Earth Radiation Budget Data, J. Climate, 19, 39-52, doi:10.1175/JCLI3611.1, 2006.

Frisch, U.: Turbulence: The Legacy of A. N. Kolmogorov, Cambridge University Press, 1995.

Hannart, A., Dufresne, J.-L., and Naveau, P.: Why climate sensitivity may not be so unpredictable, Geophys. Res. Lett., 36, L16707, doi:10.1029/2009GL039640, 2009.

Horváth, A. and Davies, R.: Comparison of microwave and optical cloud water path estimates from TMI, MODIS and MISR, J. Geophys. Res.-Atmos., 112, D01201, doi:10.1029/2006JD007, 2007.

Imkeller, P. and v. Storch, J.-S. (Eds.): Stochastic Climate Models, Birkhäuser Verlag, Basel, Swiss, 2001.

Jiménez, J.: Intermittency in turbulence, in: Proc. 15th "Aha Huliko" a Winter Workshop, Extreme Events, 81-90, 2007.

King, M. D., Platnick, S., Hubanks, P. A., Arnold, G. T., Moody, E. G., Wind, G., and Wind, B.: Collection 005 Change Summary for the MODIS Cloud Optical Property (06_OD) Algorithm, available at: http://modis-atmos.gsfc.nasa.gov/products_ C005update.html, last access: 2010, 2006.

Kostinski, A. and Shaw, R.: Scale-dependent droplet clustering in turbulent clouds, J. Fluid Mech., 434, 389-398, 2001.

Krijger, J. M., van Weele, M., Aben, I., and Frey, R.: Technical Note: The effect of sensor resolution on the number of cloud-free observations from space, Atmos. Chem. Phys., 7, 2881-2891, doi:10.5194/acp-7-2881-2007, 2007.

Liu, Y. and Daum, P.: Spectral dispersion of cloud droplet size dis- tributions and the parameterization of cloud droplet effective radius, Geophys. Res. Lett., 7, 1903-1906, 2000.

Liu, Y. and Hallet, J.: On size distributions of droplets growing by condensation: A new conceptual model, J. Atmos. Sci., 55, 527536, 1998.

Liu, Y., Daum P., and Hallet, J.: A Generalized Systems Theory for the Effect of Varying Fluctuations on Cloud Droplet Size Distributions, J. Atmos. Sci., 59, 2279-2289, 2002.

Lovejoy, S.: The Area-Perimeter Relation for Rain and Cloud Areas, Science, 216, 185-187, 1982.

Lovejoy, S., Schertzer, D., Allaire, V., Bourgeois, T., King, S., Pinel, J., and Stolle, J.: Atmospheric complexity or scale by scale simplicity?, Geophys. Res. Lett., 36, L01801, doi:10.1029/2008GL035863, 2009.

Monin, A. and Yaglom, A.: Statistical Fluid Mechanics, M.I.T. Press, Cambridge, MA, USA, 1975.

Newman, W., Lew, J., Siscoe, G., and Fovell, R.: Systematic effects of randomness in radiative transfer, J. Atmos. Sci., 52, 427-435, 1995.

Oreopoulos, L. and Davies, R.: Plane Parallel Albedo Biases from Satellite Observations. Part I: Dependence on Resolution and Other Factors, J. Climate, 11, 919-932, 1998.

Oreopoulos, L., Platnick, S., Hong, G., Yang, P., and Cahalan, R. F.: The shortwave radiative forcing bias of liquid and ice clouds from MODIS observations, Atmos. Chem. Phys., 9, 5865-5875, doi:10.5194/acp-9-5865-2009, 2009.

Pierrehumbert, R.: Anomalous scaling of high cloud variability in the tropical Pacific, Geophys. Res. Lett., 23, 1095-1098, 1996.

Platnick, S., King, M., Ackerman, S., Menzel, W., Baum, B., Riedl, C., and Frey, R.: The MODIS cloud products: Algorithms and examples from Terra, IEEE T. Geosci. Remote., Aqua Special Issue, 41, 459-473, 2003.

Pointikis, C. and Hicks, E.: Contribution to the cloud droplet effective radius parameterization, Geophys. Res. Lett., 19, 227-2230, 1992.

Pujol, T.: Eddy Heat Diffusivity at Maximum Dissipation in a Radiative-convective One-dimensional Climate Model, J. Meteorol. Soc. Jpn., 81, 305-315, 2003.

Quaas, J., Ming, Y., Menon, S., Takemura, T., Wang, M., Penner, J. E., Gettelman, A., Lohmann, U., Bellouin, N., Boucher, O., Sayer, A. M., Thomas, G. E., McComiskey, A., Feingold, G., Hoose, C., Kristjánsson, J. E., Liu, X., Balkanski, Y., Donner, L. J., Ginoux, P. A., Stier, P., Grandey, B., Feichter, J., Sednev, I., Bauer, S. E., Koch, D., Grainger, R. G., Kirkevåg, A., Iversen, T., Seland, Ø., Easter, R., Ghan, S. J., Rasch, P. J., Morrison, H., Lamarque, J.-F., Iacono, M. J., Kinne, S., and Schulz, M.: Aerosol indirect effects - general circulation model intercomparison and evaluation with satellite data, Atmos. Chem. Phys., 9, 8697-8717, doi:10.5194/acp-9-8697-2009, 2009.

Roe, G. and Baker, M.: Why Is Climate Sensitivity So Unpredictable?, Science, 318, 629-632, 2007.

Rossow, W., Delo, C., and Cairns, B.: Implications of the Observed Mesoscale Variations of Clouds for the Earth's Radiation Budget, J. Climate, 15, 557-585, 2002.

Schlosser, C. A. and Houser, P. R.: Assessing a Satellite-Era Perspective of the Global Water Cycle, J. Climate, 20, 1316-1338, doi:10.1175/JCLI4057.1, 2007.

Schutgens, J. and Roebeling, R. A.: Validating the Validation: The Influence of Liquid Water Distribution in Clouds on the In- 
tercomparison of Satellite and Surface Observations, J. Atmos. Ocean. Technol., 26, 1457-1474, 2009.

Sellers, W.: A climate model based on the energy balance of the earth-atmosphere systems, J. Appl. Meteorol., 8, 392-400, 1969.

Shenk, W. and Salomonson, V.: A simulation study exploring the effects of sensor spatial resolution on estimates of cloud cover from satellites, J. Appl. Meteorol., 11, 214-220, 1972.

Solomon, S.: Climate Change 2007: The physical science basis., Tech. rep., International Panel on Climate Change, Cambridge, UK, 2007.
Trenberth, K. E., Stepaniak, D. P., and Caron, J.: Accuracy of atmospheric energy budgets, J. Climate, 15, 3343-3360, 2003.

Wood, R. and Hartmann, D. L.: Spatial Variability of Liquid Water Path in Marine Low Cloud: The Importance of Mesoscale Cellular Convection, J. Climate, 19, 1748-1764, doi:10.1175/JCLI3702.1, 2006. 